\title{
Recognizing the emotional valence of names: An ERP study
}

\author{
Lin Wang ${ }^{\mathrm{a}, *}$, Zude Zhu ${ }^{\mathrm{b}}$, Marcel Bastiaansen ${ }^{\mathrm{c}, \mathrm{d}}$, Peter Hagoort ${ }^{\mathrm{c}, \mathrm{d}}$, Yufang Yang ${ }^{\mathrm{a}, *}$ \\ ${ }^{a}$ Key Laboratory of Behavioral Science, Institute of Psychology, Chinese Academy of Sciences, China \\ ${ }^{\mathrm{b}}$ Center for the Study of Applied Psychology, South China Normal University, China \\ ${ }^{\mathrm{c}}$ The Neurobiological of Language Group, Max Planck Institute for Psycholinguistics, Netherlands \\ ${ }^{\mathrm{d}}$ Radboud University Nijmegen, Donders Institute for Brain, Cognition and Behaviour, Netherlands
}

\section{A R T I C L E I N F O}

\section{Article history:}

Accepted 14 January 2013

\section{Keywords:}

Emotional valence

Common nouns

Proper names

Recognition

ERP

\begin{abstract}
A B S T R A C T
Unlike common nouns, person names refer to unique entities and generally have a referring function. We used event-related potentials to investigate the time course of identifying the emotional meaning of nouns and names. The emotional valence of names and nouns were manipulated separately. The results show early N1 effects in response to emotional valence only for nouns. This might reflect automatic attention directed towards emotional stimuli. The absence of such an effect for names supports the notion that the emotional meaning carried by names is accessed after word recognition and person identification. In addition, both names with negative valence and emotional nouns elicited late positive effects, which have been associated with evaluation of emotional significance. This positive effect started earlier for nouns than for names, but with similar durations. Our results suggest that distinct neural systems are involved in the retrieval of names' and nouns' emotional meaning.
\end{abstract}

Crown Copyright @ 2013 Published by Elsevier Inc. All rights reserved.

\section{Introduction}

The distinct use of common nouns and proper names has an evolutionary advantage (Müller \& Kutas, 1996; Semenza, 2006). For instance, common nouns allow for an efficient warning by using categorical labels to mark entities (e.g., calling 'snakes' for a kind of thin, long and legless animal), whereas proper names serve similar warning functions by calling a specific name of the individual in danger (e.g., calling 'Bill' for the youngest child). Person name is a typical kind of proper name (Hollis \& Valentine, 2001).

Common nouns and person names differ from each other in several aspects. First, common nouns refer to a class of objects while proper names refer to unique entities (Semenza \& Zettin, 1989). For example, the common noun 'snake' represents a class of snakes and it can refer to any one, while a person name 'Albert Einstein' normally refers to only one of the kinds. Second, common nouns intrinsically have meanings and imply attributes, whereas it is controversial whether names intrinsically carry any meaning. Kripke (1981) pointed out that a name does not carry meaning as it only identifies an individual without providing any attribute. In contrast, Sciarone (1967) proposed that the associated description (e.g., Albert Einstein as a famous physicist) constitutes the meaning

* Corresponding authors. Address: Institute of Psychology, Chinese Academy of Sciences, Chaoyang District, Lincui Road 16, Beijing 100101, China. Tel.: +86 10 64864012; fax: +8610 64872070 (L. Wang), tel.: +8610 64888629; fax: +86 10 64872070 (Y. Yang).

E-mail addresses: wangl@psych.ac.cn (L. Wang), yangyf@psych.ac.cn (Y. Yang). of a name, which differs from the linguistic (lexical) meaning of a noun.

The difference between these two categories of words raises the question of whether they are represented or processed differently in the human brain. Empirical studies suggest a difference between them. For instance, the reaction time for names was found to be faster than for nouns both in a category decision task (Müller, 2010; Yen, 2006) and in a semantic association task (Proverbio, Mariani, Zani, \& Adorni, 2009), but to be slower in a phonological decision task (Proverbio, Lilli, Semenza, \& Zani, 2001). The difference seems to indicate that for names, categorical judgment (which entails word recognition in comprehension) is easier whereas the phonological retrieval (which entails word retrieval in production) is more demanding compared to nouns.

Further evidence for the processing difference between names and nouns comes from neuropsychological studies. Neurological damage can cause a double dissociation (only proper names are disturbed while common names are unaffected or vice versa) between retrieval or recognition of names and nouns at different levels of processing, such as phonological retrieval, semantic access and application of syntactic rules (for a review see Semenza (2009)). Both neuropsychological and neuroimaging studies have indicated that proper name processing requires the involvement of a large neural network (e.g., temporal cortex and ventro-medial prefrontal cortex), and the exact location is still a matter of debate (for reviews on this issue, see Semenza (2006, 2009, 2011)).

The different processing between names and nouns has also found support in several ERP studies. During auditory sentence 
comprehension, Müller and Kutas (1996) reported larger N1 and P2 amplitudes for names than for nouns, which according to the authors could be caused by differences in their inherent phonetic features. For instance, the phoneme $[x]$ can be found more often in common nouns than in person names. In a word retrieval experiment, Proverbio et al. (2001) presented short, written, unequivocal definitions of names and nouns to participants. The participants were asked to silently retrieve the defined words in order to perform a phonological decision task. The results showed that the retrieval of names elicited larger N1 and P3 than nouns. Recently, Proverbio et al. (2009) employed a semantic association task where the participants were asked to judge the semantic relatedness between two sequentially presented words (e.g., 'Woody' vs. 'Allen', 'social' vs. 'security'). Although similar N400 effects were found between names and nouns in response to the semantic relatedness, names elicited smaller $\mathrm{N} 400$ amplitudes than nouns regardless of the semantic relatedness. Overall, the processing difference between names and nouns could occur at any stage depending on the stimuli and task. Since names and nouns differ in many aspects, it is difficult to directly compare their processing. However, both names and nouns convey emotional meaning, which makes the emotional variable ideally suitable for studying the processing difference between names and nouns.

Current word recognition models have mainly dealt with the processing of common nouns, adjectives or verbs (e.g., Coltheart, Rastle, Perry, Langdon, \& Ziegler, 2001; Grainger \& Holcomb, 2009; Plaut, McClelland, Seidenberg, \& Patterson, 1996). These models are used to describe how orthographic and phonological information cooperate in order to access semantic information of the words. Various factors have been shown to influence this process, such as word length, word frequency, concreteness and imageability. Surprisingly, none of these models has taken emotional variables into consideration. Emotional information allows for rapid and privileged access due to its intrinsic significance, i.e., potentially threatening or rewarding stimuli are biologically relevant to species survival (Lang, Bradley, \& Cuthbert, 1997). The priority of emotional information processing has been demonstrated in both behavioral and ERP studies (for reviews see Vuilleumier (2005) and Vuilleumier and Huang (2009)). ERP technique provides us with an excellent tool to measure the time course of different types of information activated in word reading (e.g., Sereno \& Rayner, 2003). It has been shown, although not always consistently, that emotional meaning can be processed automatically at a very early stage, as indicated in some early ERP differences (such as N1, P1 and early posterior negativity) between the emotional and neutral information (for a review, see Citron (2012)). Although the exact underlying processes (visual process, selective attention or lexical activation) remain unclear, such early effects suggest that the emotional feature of words can be identified very rapidly. Besides these early effects, another ERP component that is modulated by emotional words is a late positive complex (LPC), peaking between 500 and $800 \mathrm{~ms}$, with a centroparietal distribution. Its amplitude has been found to be larger for both positive and negative words (Frischen, Eastwood, \& Smilek, 2008; Hinojosa, Méndez-Bértolo, \& Pozo, 2010), only for positive words (Herbert, Kissler, Junghöfer, Peyk, \& Rockstroh, 2006), or only for negative words (Bernat, Bunce, \& Shevrin, 2001; Kanske \& Kotz, 2007). The LPC presumably reflects a less automatic evaluation of the emotional valence.

The ERP studies on emotional valence were primarily concerned with common nouns, adjectives and verbs, with the emotional processing of names not being taken into consideration so much. Neuropsychological and neuroimaging studies have shown that name processing activated right hemisphere in the brain, which has been related to the processing of emotion. This, according to the authors, might be due to the fact that information associated with familiar names provokes an emotional reaction in the individual (Damasio, Tranel, Grabowski, Adolphs, \& Damasio, 2004; Ohnesorge \& Van Lancker, 2001; Van Lancker, 1991; Van Lancker \& Ohnesorge, 2002). Given the referential nature of names, the emotional information that is activated by a name can only be derived from the characteristics of the name bearer. So far no study has directly studied the emotional valence of names. Therefore, it remains an open question when the emotional meaning carried by names can be identified.

In order to account for the difference between names and nouns, Valentine, Moore, and Brédart (1995) proposed a model of name processing based on Bruce and Young's (1986) face recognition model and Morton's (1969) word recognition model. In this model, the initial processing of names involves the analysis of an input code that is similar to ordinary word recognition. After this initial word form analysis, name recognition units which are equivalent to face recognition units are activated if the presented name is familiar. Then the person identity node (i.e., a multimodal representation of the name bearer) and identity-specific information (e.g., the occupation or the emotional valence associated with the name bearer) are activated. Therefore, the access of a name's semantic information takes place after name recognition and person identification, which in turn are subsequent to word recognition, whereas a noun's meaning can be accessed directly from the word recognition units. The model further implies that there is only a single connection between a name and its referential meaning, whereas multiple connections exist during the retrieval of noun. For instance, the proper name Baker is connected to semantic information only via lexical nodes for a known individual with the family name Baker. It does not have a set of connections representing information about this name, such as Baker is an English name. Nevertheless, the noun baker as a common noun is connected to a large number of nodes representing semantic information about bakers, such as bakes bread and wears white uniforms. Moreover, Tacikowski, Jednoróg, Marchewka, and Nowicka (2011) attempted to map the processing stages postulated by the model with ERP components. They proposed that word form analysis is associated with $\mathrm{N} 170$, name recognition is related to N250, while person identification and semantic information activation are linked to N400 or P300. Based on this model, we hypothesize that the emotional valence of names can only be activated after identity-specific information is available, which should then be reflected by late ERP components such as N400 or P300. Nevertheless, given the behavioral significance, the emotional meaning should be identified rapidly in both person names and common nouns.

The current study aims to examine the temporal characteristics of emotional processing in names and nouns. We manipulated the emotional valence of names and nouns separately and measured the ERPs elicited by the words in each condition. Since no direct measure of name frequency is available in Chinese corpus, it is difficult to match the names and nouns for their frequency. However, it has been shown that familiarity and frequency are highly correlated (Balota, Pilotti, \& Cortese, 2001), so we matched the familiarity of names and the frequency of nouns between different emotional valence conditions. Moreover, since we are mainly interested in comparing the retrieval of emotional meaning between names and nouns, we only compared the effects caused by emotional valence of names and nouns. Based on previous studies, we expect to find both early ERP effects (such as P1, N1, P2 and EPN) and late positive effect for nouns. Nevertheless, it remains an open question as to the ERP effects elicited by names. Under the name processing model (Valentine et al., 1995), no early ERP effect is expected because the emotional meaning of a name can only be available after word recognition. Alternatively, early ERP effects would be generated if the emotional significance can be 
recognized automatically, as for nouns. In any case, we expect to obtain late positive effects for names and for nouns as an indicator of emotional evaluation. The results will provide further evidence on the automaticity of emotional information processing, which has implications for models of word recognition. Moreover, the comparison between names and nouns will shed light on the physiological differences in processing words that stand for certain individuals (i.e., names) as opposed to those that stand for categories of objects (i.e., nouns).

\section{Materials and methods}

\subsection{Participants}

Twenty six university students (mean age 21.3 years, 1824 years old; 11 males) served as paid volunteers. They were all right-handed native speakers of Chinese with normal or corrected to normal vision. None of them had dyslexia or any neurological impairment. They signed a written consent form before the EEG experiment. The data of four participants (two males) were excluded due to excessive artifacts. The final set of participants therefore consisted of 22 participants (mean age 21.5 years, range 1924; nine males).

\subsection{Stimuli}

We collected 303 person names and 350 nouns from Chinese web pages. The names included both Chinese names which consist of first and last names as well as foreign names which are the translated last names. Although Chinese names differ from western names in many aspects (e.g., the orthographic feature, the number of syllables, and the order of family and given names), the emotional meaning that is conveyed by names should not differ between them. All of the names have no more than five Chinese characters, while all the nouns are two-character Chinese words. Due to the large amount of words, these words were assigned to four lists (two lists of names with 151 or 152 names per list, and two lists of nouns with 175 nouns per list) and were then rated by 60 raters (different from the EEG participants; 15 raters per list). The familiarity of the names, the concreteness of the nouns, as well as the valence and arousal of both names and nouns were rated on 7-point Likert scales (seven indicates the most familiar, the most concrete, the most positive, and the most arousing).

\subsubsection{Selection of person names}

We intended to select names that are familiar to the participants but differ in valence ratings. Therefore, we first selected the names whose familiarity scores were larger than 3.4. Then we categorized the names into three groups based on their valence scores: negative (valence rating $<4$ ), neutral (valence rating between 4 and 5) and positive (valence rating $>5$ ), with 38 names per group. A statistical analysis confirmed that the three groups differed in their valence scores: $F_{(2,74)}=337.53, p<.001$; negative vs. neutral: $t_{(37)}=-13.11, p<.001$; negative vs. positive: $t_{(37)}=$ $-26.09, p<.001$; neutral vs. positive: $t_{(37)}=-12.92, p<.001$; mean $\pm \mathrm{SD}=2.91 \pm .55,4.30 \pm .37,5.32 \pm .31$ respectively for the negative, neutral and positive words. The negative, neutral and positive names were matched for the number of strokes (a stroke is a movement of a writing instrument on a writing surface): $F_{(2,74)}<1$; mean $\pm \mathrm{SD}=21.29 \pm 7.40,21.34 \pm 6.18,20.11 \pm 5.38$, respectively. The positive names were rated as being more familiar than the neutral and negative names: $F_{(2,74)}=13.53, p<.001$; positive vs. negative: $t_{(37)}=4.20, p<.001$; positive vs. neutral: $t_{(37)}=4.79$, $p<.001$; negative vs. neutral: $t_{(37)}=1.08, p=.29$; mean $\pm \mathrm{SD}=$ $4.98 \pm .57,4.85 \pm .53,5.41 \pm .44$, respectively for the negative, neutral and positive words. Besides, the arousal ratings of the names showed a similar difference pattern as the valence ratings: $F_{(2,74)}=51.91 \quad(p<.001)$, negative vs. neutral: $t_{(37)}=$ $-2.31, p=.027$; negative vs. positive: $t_{(37)}=-9.71, p<.001$; neutral vs. positive: $t_{(37)}=-7.71, \quad p<.001 ; \quad$ mean $\pm \mathrm{SD}=3.86 \pm .08$, $4.10 \pm .07,4.86 \pm .07$ respectively for the negative, neutral and positive names. Across the three valence groups, these names were matched for occupation (politician, entertainment, fictional or scholar), sex (male or female) and epoch of popularity (past, recent or current) of the personalities, so that each condition contains equal number of names that belong to males or females with different occupations who were famous in either past, recent or current days (see Supplementary Table 1 for a list of names).

\subsubsection{Selection of common nouns}

Based on the valence scores, we selected 40 negative (valence rating < 4.3), 40 neutral (valence rating between 4.3 and 5) and 40 positive (valence rating $>5$ ) nouns that showed differences in the valence rating: mean $\pm \mathrm{SD}=1.97 \pm .38,4.30 \pm .31$ and $5.55 \pm .30$, respectively. A statistical analysis performed on the valence scores confirmed the difference: $F_{(2,78)}=1117.81, p<.001$; negative vs. neutral: $t_{(39)}=-26.19, p<.001$; negative vs. positive: $t_{(39)}=-49.39, \quad p<.001$; neutral vs. positive: $t_{(39)}=-18.53$, $p<.001$. They were matched for log-frequency, which was obtained from a Chinese corpus developed by Cai and Brysbaert (2010): $F_{(2,78)}<1$, mean $\pm \mathrm{SD}=2.54 \pm .53,2.54 \pm .57,2.48 \pm .44$, respectively for the negative, neutral and positive words. They were also matched for concreteness $\left(F_{(2,78)}<1\right.$, mean $\pm \mathrm{SD}=$ $3.24 \pm .63,3.18 \pm .48,3.12 \pm .52$, respectively) and the number of strokes $\quad\left(F_{(2,78)}<1, \quad\right.$ mean $\pm \mathrm{SD}=17.58 \pm 5.88, \quad 16.15 \pm 4.41$, $17.05 \pm 5.08$, respectively). The arousal ratings of the nouns showed a similar pattern as the valence ratings: negative $<$ neutral < positive; $F_{(2,78)}=85.88(p<.001)$; negative vs. neutral: $t_{(39)}=$ $-2.34, p=.025$; negative vs. positive: $t_{(39)}=-11.40, p<.001$; neutral vs. positive: $t_{(39)}=-12.91, p<.001 ;$ mean $\pm \mathrm{SD}=3.58(.59$, $3.86 \pm .45,4.91 \pm .41$, respectively for the negative, neutral and positive nouns (see Supplementary Table 2 for a list of nouns).

\subsection{Procedure}

Participants were seated in a comfortable chair in front of a computer screen. The words were presented in white color on a black background, with a font size of 48 . A trial started with a fixation cross (duration $2000 \mathrm{~ms}$ ) in the center of the screen followed by a $300 \mathrm{~ms}$ blank screen. Then the word was presented for $1000 \mathrm{~ms}$. After a $300 \mathrm{~ms}$ blank screen interval, an instruction appeared on the screen asking the participants to judge the valence of the presented word within $3000 \mathrm{~ms}$. The participants were instructed to press the key on the number keyboard: ' 1 ', ' 2 ' and ' 3 ' to be pressed by the right index, middle and ring finger signaling negative, neutral and positive valence (or positive, neutral and negative valence), respectively. The mapping of valence to keys was counterbalanced across participants. The next trial began $300 \mathrm{~ms}$ after the response. For those names that were unknown to the participants, the participants were instructed to press the same key (' 2 ' on the number keyboard) as for the neutral names. These unknown names were then identified from the full list of names after the EEG experiment. They were told not to move or blink during the presentation of words, but to blink during the presentation of the fixation cross.

As our primary interest is the access of names' emotional meaning, the 114 names were presented preceding the 120 nouns in order to avoid any spillover effect from the nouns. The stimuli were divided into six blocks in total (three blocks of 38 names, followed by three blocks of 40 nouns), with each block lasting about 4 min. The words were presented in a pseudo-random order, with no 
more than three words of the same condition being presented in succession. In between blocks there was a small break, after which subjects could start the next block by pressing a button. The whole experiment took about $1 \mathrm{~h}$, including subject preparation, instructions and a short practice consisting of nine words.

\subsection{Electroencephalogram (EEG) recording and analysis}

The data were recorded with a 64-channel NeuroScan system (10-20 system). A vertex electrode served as the reference, and a forehead electrode served as the ground. The vertical (VEOG) and horizontal (HEOG) eye movements were monitored through four electrodes placed around the orbital region (bipolar montage). All electrode impedances were kept below $5 \mathrm{~K} \Omega$ during the experiment. Recording was done with a band pass filter of $0.05-200 \mathrm{~Hz}$ and a sampling rate of $1000 \mathrm{~Hz}$.

The EEG data were re-referenced off-line to the average of both mastoids. The VEOG artifacts were automatically corrected by NeuroScan software (Semlitsch, Anderer, Schuster, \& Presslich, 1986). Data were filtered off-line with a $0.01-30 \mathrm{~Hz}$ ( $24 \mathrm{~dB} /$ oct slope) band-pass filter. Critical epochs ranged from $100 \mathrm{~ms}$ before to $1000 \mathrm{~ms}$ after the onset of the words, with $100 \mathrm{~ms}$ before the onset serving as the baseline. An automatic artifact rejection procedure was taken to exclude trials exceeding $\pm 90 \mu \mathrm{V}$. Additionally, the trials containing unknown names were manually excluded. In the end, four participants (two males) were excluded because the total number of rejected trials exceeded 30 (see also Section 2.1).

\subsection{Condition re-assignment of the words}

We calculated the percentage of the "correct" response where the responses of the EEG participants corresponded to the conditions obtained from the ratings. The results showed low correspondence between the response and the condition for both names (mean $\pm \mathrm{SD}=60.37 \% \pm 5.13 \%$ ) and nouns (mean $\pm \mathrm{SD}=81.48 \% \pm$ $9.46 \%$ ). This suggests that the participants of the EEG experiment made different judgments on the valence of the words compared to the initial raters. This was even more evident for names than nouns. The discrepancy further suggests that names have more arbitrary associations with emotional valence than nouns. The low consistency for the two groups of participants might be ex- plained by a large variability in participants' judgments of the words. In order to test this possibility, we calculated the standard deviation of the ratings of the initial raters and the responses of the EEG participants. We found large standard deviations for both the raters (mean $\pm \mathrm{SD}$ of the standard deviation $=1.39 \pm 0.29$ and $1.01 \pm 0.28$ on a 7-point scale, respectively for names and nouns) and the EEG participants (mean \pm SD of the standard deviation $=0.57 \pm 0.29$ and $0.32 \pm 0.21$ on a 3 -point scale, respectively for names and nouns). Since different scales were adopted in the EEG experiment (a 3-point scale) from the ratings (a 7-point scale), we converted the 7-point scale into a 3 -point scale by re-coding ratings of 1,2 and 3 as 1 , rating of 4 as 2, and ratings of 5, 6 and 7 as 3 . Then the standard deviation of the responses was measure across all the participants (including the raters as well as the participants in the EEG experiment): mean \pm SD of the standard deviation $=0.63 \pm 0.14$ and $0.42 \pm 0.16$ respectively for the names and nouns on a 3 -point scale. The large standard deviations indicate that the actual rating score of a particular word varies greatly among participants. For instance, the neutral name whose rating score is 2 on average was actually rated by most of the participants (over $95 \%$ ) as $2 \pm 0.63 * 2$, i.e., in the range of $\sim 1$ to $\sim 3$. Therefore, the mean rating scores do not adequately reflect the participants' real judgments of the valence of the words.

In order to increase the sensitivity of the experiment, we re-assigned all words to condition based on each participant's judgments in the EEG experiment, such that the words they judged as negative were assigned to the negative condition, the words they judged as neutral were assigned to the neutral condition, and the words they judged as positive were assigned to the positive condition. We threw away a few trials (five trials and six trials respectively for the names and nouns in each condition) in order to subsequently match the words for familiarity, concreteness, frequency and the number of strokes. Then we obtained the averaged rating scores in each condition for each participant for the remaining trials. Tables 1 and 2 present the rating scores as well as the behavioral results for the re-assigned three conditions based on EEG participants' responses, for the names and nouns respectively. In the end, the ERPs were calculated by averaging over trials according to the re-arranged conditions for each electrode and each subject. Note that unequal number of trials among emotional valence conditions was resulted from this procedure. This means

Table 1

Behavioral measurements of the names in the rearranged conditions.

\begin{tabular}{|c|c|c|c|c|c|}
\hline & Negative & Neutral & Positive & $F_{(2,42)}$ & $p$ Value \\
\hline Valence & $3.07(.14)$ & $4.18(.36)$ & $4.76(.12)$ & 319.91 & $<.001$ \\
\hline Arousal & $3.94(.075)$ & $4.22(.17)$ & $4.43(.06)$ & 96.41 & $<.001$ \\
\hline Familiarity & $5.04(.06)$ & $4.98(.18)$ & $5.04(.04)$ & 2.42 & .129 \\
\hline $\mathrm{Nr} \_s t r o k e$ & $21.59(.52)$ & $21.35(1.86)$ & $21.01(.54)$ & 1.24 & .288 \\
\hline RT & $573.39(200.34)$ & $642.95(203.44)$ & $533.72(184.06)$ & 10.19 & .001 \\
\hline $\mathrm{Nr} \_t r l$ & $31(6)$ & $21(10)$ & $38(8)$ & 17.79 & $<.001$ \\
\hline
\end{tabular}

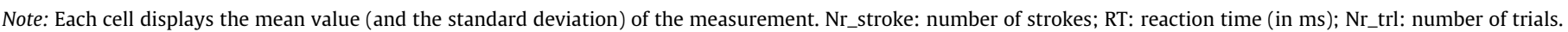

Table 2

Behavioral measurements of the nouns in the rearranged conditions.

\begin{tabular}{|c|c|c|c|c|c|}
\hline & Negative & Neutral & Positive & $F_{(2,42)}$ & $p$ Value \\
\hline Valence & $2.07(.15)$ & $4.36(.28)$ & $5.29(.16)$ & 1831.59 & $<.001$ \\
\hline Arousal & $3.61(.05)$ & $3.93(.15)$ & $4.72(.14)$ & 414.72 & $<.001$ \\
\hline Concreteness & $3.15(.05)$ & $3.12(.06)$ & $3.14(.04)$ & 1.10 & .335 \\
\hline Frequency & $2.53(.03)$ & $2.55(.08)$ & $2.51(.04)$ & 1.60 & .219 \\
\hline $\mathrm{Nr}$ _stroke & $17.15(.36)$ & $17.00(.68)$ & $16.98(.50)$ & .57 & .528 \\
\hline RT & $403.20(202.11)$ & $498.75(258.18)$ & $427.05(179.81)$ & 9.54 & .002 \\
\hline $\mathrm{Nr} \_t r l$ & $31(4)$ & $29(7)$ & $43(9)$ & 18.06 & $<.001$ \\
\hline
\end{tabular}

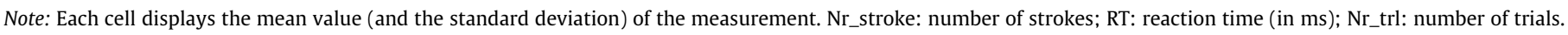


that the noise level of ERPs differs between conditions. However, since the mean amplitude is not affected by the noise level (Luck, 2010), we performed statistical analysis on the mean amplitudes of the waveforms.

\subsection{Statistical analysis}

\subsubsection{Cluster-based random permutation test}

The statistical difference between two conditions was evaluated by a cluster-based random permutation test (Maris \& Oostenveld, 2007), which was implemented in the Matlab toolbox Fieldtrip (Oostenveld, Fries, Maris, \& Schoffelen, 2011). This approach controls the Type- 1 error rate which involves multiple comparisons (one comparison for each electrode). Here is a brief description of the general procedure. First, for every data sample (electrode or electrode * time point) a simple dependent-samples $t$ test is performed. All adjacent data samples (spatial or temporal) exceeding a preset significance level (5\% here) are grouped into clusters. For each cluster the sum of the $t$ statistics is used in the cluster-level test statistic. Then a null distribution which assumes no difference between conditions is created. This distribution is obtained by 1000 times randomly assigning the conditions in subjects and calculating the largest cluster-level statistic for each randomization. Finally, the actually observed cluster-level test statistics are compared against the null distribution, and clusters falling in the highest or lowest 2.5th percentile are considered significant.

Three separate analyses were performed on N1, P2 and late positivity. On the basis of earlier studies (Bernat et al., 2001; Hofmann, Kuchinke, Tamm, Võ, \& Jacobs, 2009; Scott, O’Donnell, Leuthold, \& Sereno, 2009; van Hooff, Dietz, Sharma, \& Bowman, 2008) and visual inspection, the amplitudes of the $\mathrm{N} 1$ and $\mathrm{P} 2$ were tested for the statistical differences among conditions at their peak latencies of N1 (90-110 ms) and P2 (170-250 ms). Accordingly, the mean values of all electrodes (60 electrodes) in the selected time windows were entered into the analysis. Then an exploratory analysis of the positive effect in the remaining time interval (250-1000 ms) was performed in order to establish the time point from which the ERPs in the two tested conditions start to diverge. In this analysis, all time points in this time window (60 electrodes* 750 time points) were entered into the analysis. Because the cluster-based random permutation test only allows for pair-wise comparisons, we made the following comparisons separately for names and nouns: negative vs. neutral, positive vs. neutral and negative vs. positive. Before making these planned pair-wise comparisons, we performed interaction tests on the difference waveforms of two emotionally valenced conditions between names and nouns.

\subsubsection{Linear mixed model analysis}

As mentioned above, we removed some trials in order to match the words for familiarity, concreteness, frequency and the number of strokes. This procedure might have affected the statistical analysis. In order to make sure that only emotional valence had an effect on the observed ERP effects, we also performed a linear mixed model analysis, in which all the trials (after removing trials containing artifacts and unknown names) entered into the analysis. This approach was chosen because it deals with unbalanced data (i.e., unequal number of trials per subject after preprocessing) and optimally uses all information (including both categorical and continuous variables) and therefore affords the best statistical inferences about experimental effects and individual differences (Kliegl, Wei, Dambacher, Yan, \& Zhou, 2011). It has been successfully applied in word recognition studies (Amsel, 2011; Hauk, Davis, Ford, Pulvermüller, \& Marslen-Wilson, 2006; Laszlo \& Federmeier, 2011). For the names, the emotional valence, arousal, familiarity and number of strokes were taken as independent variables, while the independent variables of nouns included emo- tional valence, arousal, frequency, concreteness and number of strokes. We took the emotional valence as a categorical variable, and compared the emotionally valenced conditions with the neutral conditions (negative vs. neutral; positive vs. neutral). The arousal, familiarity, concreteness, frequency and number of strokes were transformed to $z$-values. In addition, based on the results of the cluster-based random permutation test, we averaged the ERP amplitudes in the time windows at the electrodes where significant differences between valence conditions were revealed (see Section 3.2), for each trial and each subject, and then took these values as dependent variables.

We used the lmer program of the lme4 package (Bates, 2010) to estimate fixed effects of the linear mixed model. This package is supplied in the $R$ system for statistical computing (version 2.15.0, R Development Core Team, 2011) under the GNU General Public License (version 2, June 1991). The two contrasts of valence conditions (negative vs. neutral and positive vs. neutral) and other independent variables as well their possible interactions were specified as fixed factors, while the subjects and the items were specified as random factors. We estimated the sensitivity of the model by checking the change in goodness of fit after adding or removing some variables. After comparing different models, the fixed effects in the best-fit model were reported in the results.

\section{Results}

\subsection{Behavioral results}

As mentioned above, we re-arranged the words into conditions according to each individual's responses. Then averaged reaction times (RTs) were obtained in each condition for each participant. Tables 1 and 2 show the RTs respectively for the names and nouns. We found that RTs were shorter for emotional than for neutral words: $F_{(2,42)}=10.19, p<.001 ; F_{(2,42)}=9.54, p<.001$; negative vs. neutral: $t_{(21)}=-3.34, p=.003 ; t_{(21)}=-3.33, p=.003$; positive vs. neutral: $t_{(21)}=-4.88, p<.001 ; t_{(21)}=-3.27, p=.004$; negative vs. positive: $t_{(21)}=1.35, p=.19 ; t_{(21)}=-1.50, p=.15$, respectively for the names and the nouns.

\subsection{ERP results}

3.2.1. Statistical results of the cluster-based random permutation test

Figs. 1 and 2 respectively show the grand average ERP waveforms evoked by the names and nouns, separately for the different valence conditions.

In the $\mathrm{N} 1$ time window $(90-110 \mathrm{~ms})$, the interaction test between names and nouns for the contrast of negative vs. neutral revealed a significant cluster $(p=.050)$, whereas no interaction was found between names and nouns for the contrasts of positive vs. neutral and negative vs. positive. Then we performed planned comparisons between emotional conditions separately for names and nouns. No significant cluster was found in any comparison for the names. In contrast, the emotional nouns elicited larger N1s than the neutral nouns: we found a significant cluster in the comparison of negative vs. neutral nouns over the posterior region $(p=.042)$ and in the comparison of positive vs. neutral nouns over the central region ( $p=.048$; see Fig. $3 \mathrm{~A})$. No difference was found between negative and positive nouns.

In the P2 time window (170-250 ms), none of the comparisons reached significance, neither for the names nor for the nouns.

In the later time window between 250 and $1000 \mathrm{~ms}$, the interaction test showed that negative nouns elicited an additional positivity relative to neutral nouns, which was larger than that for negative names in the time window of $530-620 \mathrm{~ms}(p=.043)$, but smaller between 880 and $950 \mathrm{~ms}(p=.029)$. Pair-wise 

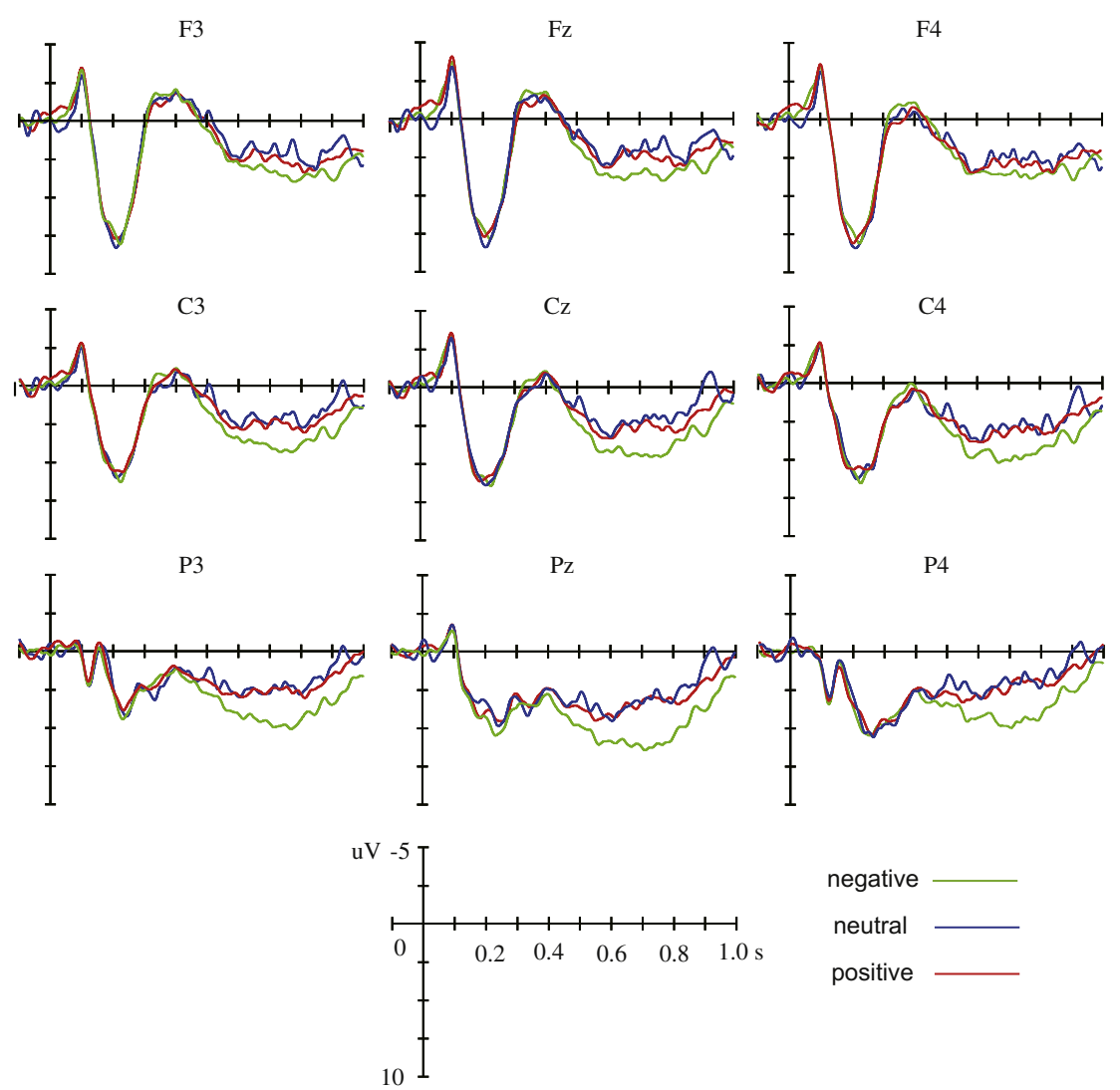

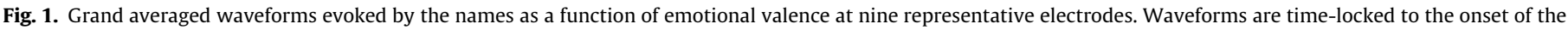
names. Negative is plotted upward.

comparisons revealed a larger positivity for the negative than for the neutral names, starting from $640 \mathrm{~ms}$ and ending at $950 \mathrm{~ms}$ over the posterior region ( $p=.002$; Fig. 3B). Similarly, the negative nouns elicited a larger positivity than the neutral nouns in the time window of $440-770 \mathrm{~ms}$ with a broad distribution $(p=.001$; Fig. 3B). In the comparison between the positive and neutral words, no interaction was found between the names and the nouns in this contrast. However, planned comparisons showed that there was a positive shift for the nouns between 500 and $600 \mathrm{~ms}$ over the right posterior region ( $p=.050$; Fig. 3B), but there was no such effect for the names. For the comparison between negative and positive words, the interaction test indicated that the nouns elicited a larger positive effect than the names between 507 and $629 \mathrm{~ms}$ $(p=.036)$. Further comparisons showed significantly larger positivity for the negative than the positive words for both names $(p=.014$, in the time window of $640-810 \mathrm{~ms})$ and nouns $(p<.001$, in the time window of 505-803 ms) over the posterior region (Fig. 3B).

\subsubsection{Statistical results of the linear mixed model analysis}

Based on the results obtained from the cluster-based random permutation test, five linear mixed models were tested for the observed ERP effects (the $\mathrm{N} 1$ and late positive effects). The dependent variables of the models were obtained from the averaged ERP amplitudes of the significant clusters (combinations of time windows and electrodes, which were indicated in Fig. 3). The best-fit linear mixed models included only the emotion valence as a fixed effect, and the items as a random effect. Adding other variables or interactions to the models did not improve, if not deteriorate, the fit of the models. The significant effects of emotional valence were in line with the effects revealed in the cluster-based random permutation test, except that the $\mathrm{N} 1$ effect between the positive and neutral nouns failed to be significant ( $p=.19$ ). The corresponding effects obtained from the two analyses further confirmed our findings.

\section{Discussion}

This study aimed to investigate the time course of emotional processing in both names and nouns. The ERP effects in response to emotional valence were separately assessed for names and nouns based on individual responses. Strikingly, emotional valence produced an $\mathrm{N} 1$ effect (between 90 and $110 \mathrm{~ms}$ ) for nouns only, which was more evident for the negative nouns. In addition, late positive effects were observed for both names and nouns. For names, we found a larger positivity elicited by the negative than the neutral names in the time interval of $640-950 \mathrm{~ms}$. For nouns, both the negative and the positive nouns elicited larger positivities than the neutral nouns in the time windows of $440-770 \mathrm{~ms}$ and 500-600 ms respectively.

\subsection{An early N1 effect was only found for nouns}

The N1 effect has been reported in previous studies on emotional words processing (Bernat et al., 2001; Hofmann et al., 2009; Scott, O'Donnell, Leuthold, \& Sereno, 2009; van Hooff et al., 2008). This early effect might reflect enhanced perceptual processing induced by the salience of emotional words (especially the negatively valenced words). The strong association between the emotional salience and the word form allows for a quick and coarse analysis of the words, before detailed semantic processing takes place. The early N1 effects elicited by the high frequency nouns indicate that very salient emotional stimuli are easily processed, even before the access of lexical information. As mentioned in 

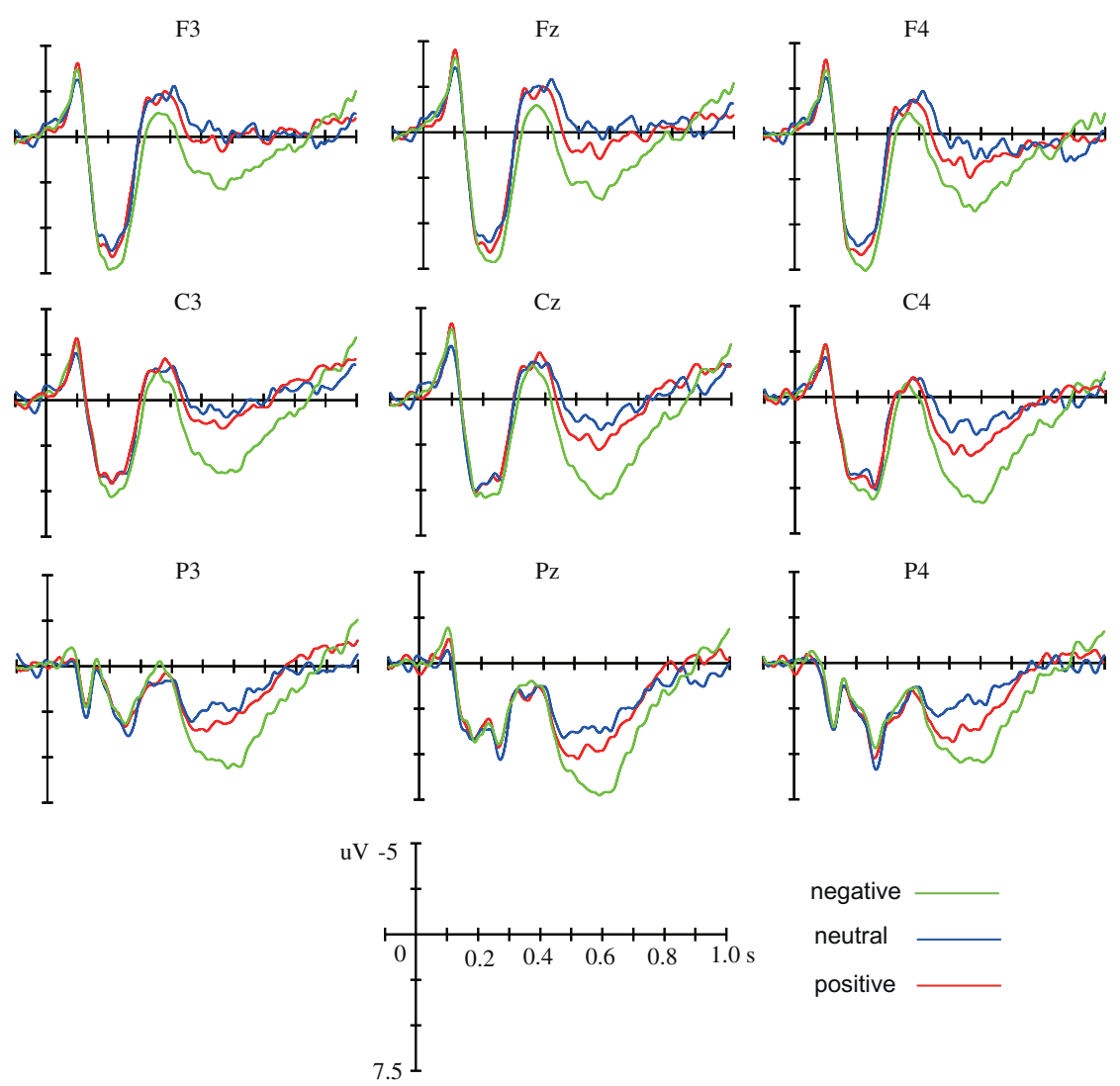

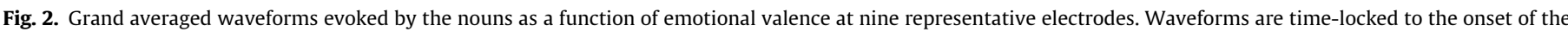
nouns. Negative is plotted upward.

the introduction, current word recognition models have rarely taken emotional variables into consideration (Coltheart et al., 2001; Grainger \& Holcomb, 2009; Plaut et al., 1996). This makes it difficult to interpret our findings in terms of these models. However, some studies have attempted to study the interaction between emotional valence and lexical variables, such as word frequency, word concreteness and grammatical class. For instance, Scott, O'Donnell, Leuthold, and Sereno (2009) and Kissler, Herbert, Peyk, and Junghofer (2007) found that the early ERP effects in response to emotional valence was only observed for high frequency words. In addition, Kanske and Kotz (2007) found that the late positive effect was revealed for concrete words but not for abstract words. Moreover, Palazova, Mantwill, Sommer, and Schacht (2011) reported that the activation of emotional meaning depends on the grammatical class of words. They have shown that emotion effect occurred approximately at the same time as frequency effect takes place for nouns and adjectives, whereas the emotion effect followed the frequency effect for verbs. Overall, although the time locus of emotional effect in relation to lexical access is still unclear, these findings suggest that emotional variables contribute to word recognition in addition to other lexical and semantic variables.

However, another category of words, names, elicited no such early effect in response to emotional valence. This is in line with the name processing model proposed by Valentine et al. (1995). According to the model, the emotional meaning of names can only be accessible after word recognition, which takes place around 200 ms (Cohen et al., 2000; Dien, 2009). This model also received support from other studies (Schweinberger, Pickering, Burton, \& Kaufmann, 2002; Tacikowski et al., 2011; Valentine, Bredart, Lawson, \& Ward, 1991; Valentine et al., 1995). In the study of Valentine et al. (1991), they found that in tasks which did not require access to the identity-specific information (e.g., nationality decision and name articulation), the RT of names with high frequency was shorter than those with low frequency, which was analogous to the effect of word frequency. On the contrary, in tasks which did require access to the identity-specific information (e.g., familiarity decision and semantic classification), the RT was longer for names with high frequency than for names with low frequency, which was analogous to the effect of distinctiveness in face recognition. In addition, Valentine et al. (1995) used surnames of celebrities that are English words (e.g., "Bush" and "Wood") to explore the relationship between production of common nouns and person names that share the same phonology. They found that the access to noun lexicon had no priming effect on the person recognition, whereas the access to the identity information of names primed the recognition of common nouns. The results suggest that word recognition precedes the activation of identity-specific information. Using ERP technique, Schweinberger et al. (2002) and Tacikowski et al. (2011) both found that previously exposed names resulted in a late positive shift after $300 \mathrm{~ms}$ relative to previously unexposed names, reflecting a change in post-perceptual representations of names.

In the current study, the apparent difference between names and nouns reflects their different ways of linking to emotional meaning. For nouns, a direct link was established between the emotional meaning and the visual word form, whereas the access of names' emotional meaning was mediated by name recognition and person identification. The distinct early processing between names and nouns may rely on some special features of Chinese names, which were constituted by family names and given names. While the family name only represents the origin of the name bearer, the given name generally contains semantic content which 


\section{(A) N1 effect}

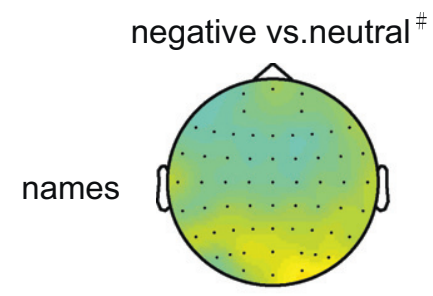

90-110ms

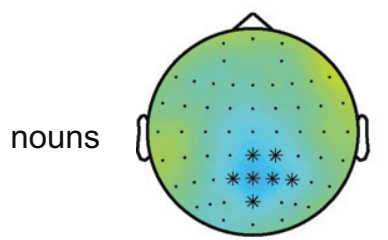

90-110ms positive vs.neutral

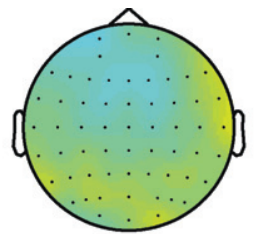

90-110ms

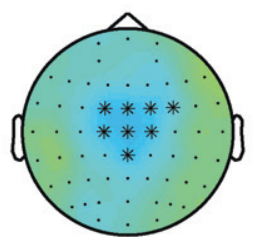

90-110ms negative vs.positive

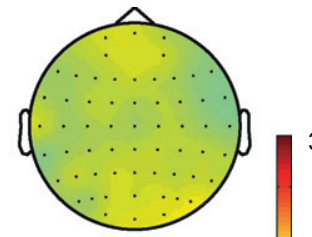

90-110ms

0

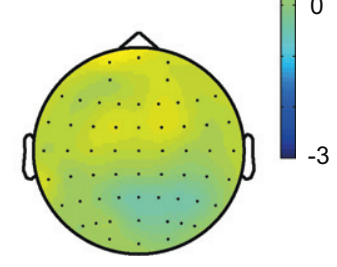

90-110ms

(B) Late positive effect

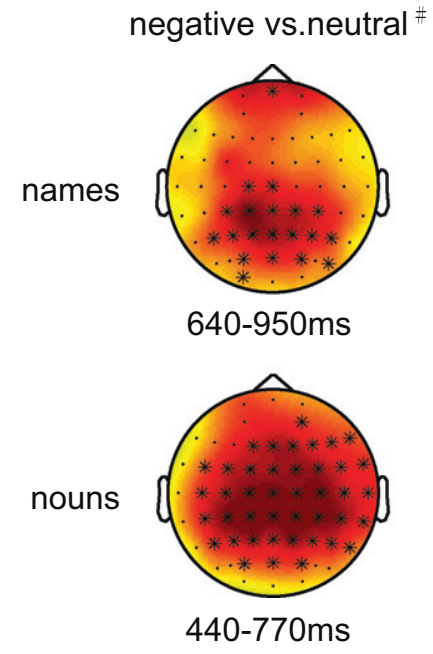

\section{positive vs.neutral}

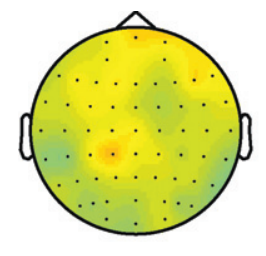

640-950ms

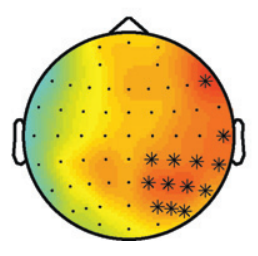

500-600ms negative vs.positive \#

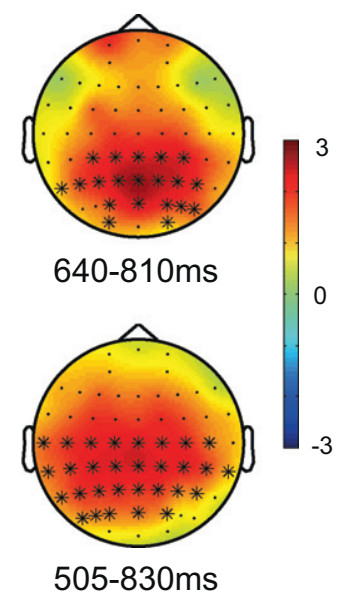

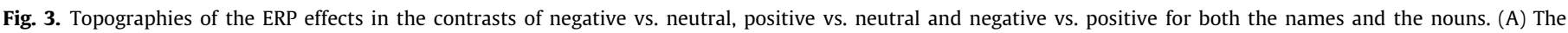

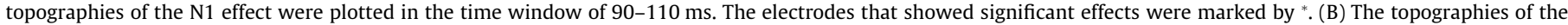

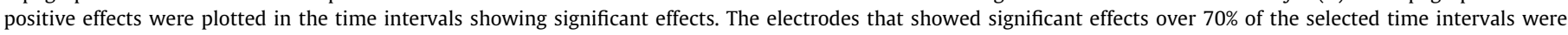
marked by *. \# Indicates a significant interaction between word category (names or nouns) and the shown contrast.

reflects pleasant characteristics such as beauty, power or wisdom. In the current study, all the names consisted of characters with positive meaning regardless of the emotional valence of the name bearers. Therefore, the initial coarse analysis did not differ among conditions for names. In contrast, the nouns with various emotional valences differ at the low-level of perception, leading to the N1 effect.

Overall, the results seem to suggest that different neural systems serve as connections between emotional meanings and different types of words (i.e., names vs. nouns).

\subsection{The late positive effect started earlier for nouns than for names}

For both names and nouns, emotional words elicited larger positivities than neutral words except for the lack of such an effect between the positive and neutral names. The positive effect elicited by emotional valence has been repeatedly reported (Bernat et al., 2001; Frischen et al., 2008; Hinojosa et al., 2010; Kanske \& Kotz, 2007). It reflects an explicit evaluation of the emotional valence of the words. In order to ensure that participants engage in emotional processing and that the emotional conditions of words more closely reflect each participant's responses, the current study employed an explicit task in which participants were explicitly asked to judge the emotional valence of words. It might be interesting to see whether the same effect can be observed in implicit tasks.

No late positive effect was found for the positively valenced names relative to neutral names. This could be due to a relatively small, although significant, difference in emotional valence between the positive and neutral names (4.76 vs. 4.18 in a 7-point scale). Another possibility is that people give greater weight to negative entities and respond stronger to the negative information than the neutral and positive information, which is also referred to as "negative bias" (Rozin \& Royzman, 2001).

The positive effects started earlier for nouns than for names (440 ms vs. $640 \mathrm{~ms}$ ). This is consistent with the RT results, where the emotional valence judgment was faster for nouns than for names. The results might reflect that the retrieval of emotional meaning of nouns was less demanding or with a more direct pathway than that of names, as suggested in the study by Proverbio et al. (2001). Note, however, it could also be due to the frequency difference as we did not match the names and nouns for this. 
Moreover, the similar durations (around $200 \mathrm{~ms}$ ) of the positive effect between nouns and names (440-770 ms vs. 640-950 ms) suggest a substantially similar process of emotional evaluation.

\section{Conclusions}

By examining ERP responses to names and nouns with different emotional valence, we have provided evidence for the physiological difference between names and nouns. We found that the emotional nouns (most evidently the negative nouns) produced both early N1 effects and late positive effects, whereas only a late positive effect was found for negative compared to neutral names. The early $\mathrm{N} 1$ effects for nouns indicate that the emotional significance of nouns can be identified very rapidly. In contrast, the lack of such $\mathrm{N} 1$ effect for names suggests that the emotional meaning of names can only be accessed after initial word recognition as well as subsequent name recognition and person identification. Moreover, the emotional evaluation occurred earlier for nouns than for names (but with similar durations), as indicated by both shorter RTs and earlier onsets of the positive effects. Overall, the results seem to suggest that distinct neural processes are involved in the retrieval of emotional meaning of names and nouns.

\section{Acknowledgments}

This work was supported by the Scientific Foundation of Institute of Psychology, Chinese Academy of Sciences [Grant Number: Y1CX312005] to Lin Wang, and the Natural Science Foundation of China [Grant Number: 31100811] to Zude Zhu.

\section{Appendix A. Supplementary material}

Supplementary data associated with this article can be found, in the online version, at http://dx.doi.org/10.1016/j.bandl.2013. 01.006 .

\section{References}

Amsel, B. D. (2011). Tracking real-time neural activation of conceptual knowledge using single-trial event-related potentials. Neuropsychologia, 49(5), 970-983. doi: 10.1016/j.neuropsychologia.2011.01.003.

Bates, D. M. (2010). Lme4: Mixed-effects modeling with R. New York: Springer.

Bernat, E., Bunce, S., \& Shevrin, H. (2001). Event-related brain potentials differentiate positive and negative mood adjectives during both supraliminal and subliminal visual processing. International Journal of Psychophysiology, 42(1), 11-34.

Balota, D. A., Pilotti, M., \& Cortese, M. J. (2001). Subjective frequency estimates for 2,938 monosyllabic words. Memory E Cognition, 29(4), 639-647.

Bruce, V., \& Young, A. (1986). Understanding face recognition. British Journal of Psychology, 77(3), 305-327.

Cai, Q., \& Brysbaert, M. (2010). SUBTLEX-CH: Chinese word and character frequencies based on film subtitles. PLOS ONE, 5(6), e10729.

Citron, F. M. M. (2012). Neural correlates of written emotion word processing: A review of recent electrophysiological and hemodynamic neuroimaging studies. Brain and Language, 122(3), 211-226.

Cohen, L., Dehaene, S., Naccache, L., Lehéricy, S., Dehaene-Lambertz, G., Hénaff, M.A., et al. (2000). The visual word form area. Brain, 123(2), 291-307.

Coltheart, M., Rastle, K., Perry, C., Langdon, R., \& Ziegler, J. (2001). DRC: A dual route cascaded model of visual word recognition and reading aloud. Psychological Review, 108(1), 204-256.

Dien, J. (2009). The neurocognitive basis of reading single words as seen through early latency ERPs: A model of converging pathways. Biological Psychology, $80(1), 10-22$.

Damasio, H., Tranel, D., Grabowski, T. J., Adolphs, R., \& Damasio, A. (2004). Neural systems behind word and concept retrieval. Cognition, 92, 179-229.

Frischen, A., Eastwood, J. D., \& Smilek, D. (2008). Visual search for faces with emotional expressions. Psychological Bulletin, 134(5), 662-676.

Grainger, J., \& Holcomb, P. J. (2009). Watching the word go by: On the time-course of component processes in visual word recognition. Language and Linguistics Compass, 3(1), 128-156.

Hauk, O., Davis, M. H., Ford, M., Pulvermüller, F., \& Marslen-Wilson, W. D. (2006). The time course of visual word recognition as revealed by linear regression analysis of ERP data. Neurolmage, 30(4), 1383-1400.
Herbert, C., Kissler, J., Junghöfer, M., Peyk, P., \& Rockstroh, B. (2006). Processing of emotional adjectives: Evidence from startle EMG and ERPs. Psychophysiology, 43(2), 197-206

Hinojosa, J. A., Méndez-Bértolo, C., \& Pozo, M. A. (2010). Looking at emotional words is not the same as reading emotional words: Behavioral and neural correlates. Psychophysiology, 47(4), 748-757.

Hofmann, M., Kuchinke, L., Tamm, S., Võ, M., \& Jacobs, A. (2009). Affective processing within 1/10th of a second: High arousal is necessary for early facilitative processing of negative but not positive words. Cognitive, Affective, $\mathcal{E}$ Behavioral Neuroscience, 9(4), 389-397.

Hollis, J., \& Valentine, T. (2001). Proper-name processing: Are proper names pure referencing expressions? Journal of Experimental Psychology: Learning, Memory, and Cognition, 27(1), 99-116.

Kanske, P., \& Kotz, S. A. (2007). Concreteness in emotional words: ERP evidence from a hemifield study. Brain Research, 1148, 138-148.

Kissler, J., Herbert, C., Peyk, P., \& Junghofer, M. (2007). Buzzwords: Early cortical responses to emotional words during reading. Psychological Science, 18 475-480.

Kliegl, R., Wei, P., Dambacher, M., Yan, M., \& Zhou, X. (2011). Experimental effects and individual differences in linear mixed models: Estimating the relation of spatial, object, and attraction effects in visual attention. Frontiers in Psychology, 1.

Kripke, S. (1981). Naming and necessity. Oxford, UK: Blackwell publishing.

Lang, P. J., Bradley, M. M., \& Cuthbert, B. N. (1997). Motivated attention: Affect, activation, and action. Mahwah, NJ: Erlbaum.

Laszlo, S., \& Federmeier, K. D. (2011). The N400 as a snapshot of interactive processing: Evidence from regression analyses of orthographic neighbor and lexical associate effects. Psychophysiology, 48(2), 176-186.

Luck, S. J. (2010). "Is It Legitimate to Compare Conditions with Different Numbers of Trials?" <http://erpinfo.org/Members/sjluck/Mean_Peak_Noise.pdf/view> Retrieved 22.05.11.

Müller, H. M. (2010). Neurolinguistic findings on the language lexicon: The special role of proper names. Chinese Journal of Physiology, 53(6), 351-358.

Müller, H. M., \& Kutas, M. (1996). What's in a name? Electrophysiological differences between spoken nouns, proper names and one's own name. NeuroReport, 8(1), 221-225.

Maris, E., \& Oostenveld, R. (2007). Nonparametric statistical testing of EEG- and MEG-data. Journal of Neuroscience Methods, 164(1), 177-190.

Morton, J. (1969). Interaction of information in word recognition. Psychological Review, 76(2), 165-178.

Ohnesorge, C., \& Van Lancker, D. (2001). Cerebral laterality for famous proper nouns: Visual recognition by normal subjects. Brain and Language, 77(2), 135-165. doi: 10.1006/brln.2000.2365.

Oostenveld, R., Fries, P., Maris, E., \& Schoffelen, J. M. (2011). FieldTrip: Open source software for advanced analysis of MEG, EEG, and invasive electrophysiological data. Computational Intelligence and Neuroscience, 2011, 156869.

Palazova, M., Mantwill, K., Sommer, W., \& Schacht, A. (2011). Are effects of emotion in single words non-lexical? Evidence from event-related brain potentials. Neuropsychologia, 49(9), 2766-2775.

Plaut, D. C., McClelland, J. L., Seidenberg, M. S., \& Patterson, K. (1996). Understanding normal and impaired word reading: Computational principles in quasi-regular domains. Psychological Review, 103(1), 56-115.

Proverbio, A. M., Lilli, S., Semenza, C., \& Zani, A. (2001). ERP indexes of functional differences in brain activation during proper and common names retrieval. Neuropsychologia, 39(8), 815-827.

Proverbio, A. M., Mariani, S., Zani, A., \& Adorni, R. (2009). How are 'Barack Obama' and 'President Elect' differentially stored in the brain? An ERP investigation on the processing of proper and common noun pairs. PLoS ONE, 4(9), e7126.

R Development Core Team (2011). R: A language and environment for statistical computing. R Foundation for Statistical Computing, Vienna, Austria. ISBN 3900051-07-0. Available from http://www.R-project.org/.

Rozin, P., \& Royzman, E. B. (2001). Negativity bias, negativity dominance, and contagion. Personality and Social Psychology Review, 5(4), 296-320.

Schweinberger, S. R., Pickering, E. C., Burton, A. M., \& Kaufmann, J. M. (2002). Human brain potential correlates of repetition priming in face and name recognition. Neuropsychologia, 40(12), 2057-2073.

Sciarone, B. (1967). Proper names and meaning. Studia Linguistica, 21(2), 73-86.

Scott, G. G., O'Donnell, P. J., Leuthold, H., \& Sereno, S. C. (2009). Early emotion word processing: Evidence from event-related potentials. Biological Psychology, 80(1), 95-104.

Semenza, C. (2006). Retrieval pathways for common and proper names. Cortex 42(6), 884-891.

Semenza, C. (2009). The neuropsychology of proper names. Mind E Language, 24(4) 347-369.

Semenza, C. (2011). Naming with proper names: The left temporal pole theory. Behavioural Neurology, 24(4), 277-284.

Semenza, C., \& Zettin, M. (1989). Evidence from aphasia for the role of proper names as pure referring expressions. Nature, 342(6250), 678-679.

Semlitsch, H. V., Anderer, P., Schuster, P., \& Presslich, O. (1986). A solution for reliable and valid reduction of ocular artifacts, applied to the P300 ERP. Psychophysiology, 23(6), 695-703.

Sereno, S. C., \& Rayner, K. (2003). Measuring word recognition in reading: eye movements and event-related potentials. Trends in Cognitive Sciences, 7(11) 489-493. doi: 10.1016/j.tics.2003.09.010.

Tacikowski, P., Jednoróg, K., Marchewka, A., \& Nowicka, A. (2011). How multiple repetitions influence the processing of self-, famous and unknown names and faces: An ERP study. International Journal of Psychophysiology, 79(2), 219-230. 
Valentine, T., Bredart, S., Lawson, R., \& Ward, G. (1991). What's in a name? Access to information from people's names. European Journal of Cognitive Psychology, 3(1), 147-176.

Valentine, T., Moore, V., \& Brédart, S. (1995). Priming production of people’s names. The Quarterly Journal of Experimental Psychology A: Human Experimental Psychology, 48A(3), 513-535.

van Hooff, J. C., Dietz, K. C., Sharma, D., \& Bowman, H. (2008). Neural correlates of intrusion of emotion words in a modified Stroop task. International Journal of Psychophysiology, 67(1), 23-34.

Van Lancker, D. (1991). Personal relevance and the human right hemisphere. Brain and Cognition, 17(1), 64-92. doi: 10.1016/0278-2626(91)90067-I.
Van Lancker, D., \& Ohnesorge, C. (2002). Personally familiar proper names are relatively successfully processed in the human right hemisphere; or, the missing link. Brain and Language, 80(2), 121-129. doi: 10.1006/brln.2001.2564. Vuilleumier, P. (2005). How brains beware: Neural mechanisms of emotional attention. Trends in Cognitive Sciences, 9(12), 585-594.

Vuilleumier, P., \& Huang, Y.-M. (2009). Emotional attention: Uncovering the mechanisms of affective biases in perception. Current Directions in Psychological Science, 18, 148-152.

Yen, H.-L. (2006). Processing of proper names in Mandarin Chinese: A behavioral and neuroimaging study. Bielefeld, Germany: Bielefeld University. 